\title{
Téoros
}

Revue de recherche en tourisme

\section{Un essai de diagnostic}

\section{Le marketing touristique au Québec}

\section{Pierre Filiatrault et Jean-Charles Chebat}

Volume 3, numéro 1, mars 1984

Le marketing touristique : perspectives et limites

URI : https://id.erudit.org/iderudit/1080814ar

DOI : https://doi.org/10.7202/1080814ar

Aller au sommaire du numéro

Éditeur(s)

Université du Québec à Montréal

ISSN

0712-8657 (imprimé)

1923-2705 (numérique)

Découvrir la revue

Citer cet article

Filiatrault, P. \& Chebat, J.-C. (1984). Un essai de diagnostic : le marketing touristique au Québec. Téoros, 3(1), 2-4. https://doi.org/10.7202/1080814ar d'utilisation que vous pouvez consulter en ligne.

https://apropos.erudit.org/fr/usagers/politique-dutilisation/ 


\section{Le marketing touristique au Québec}

\section{Un essai de diagnostic}

Pierre Filiatrault et Jean-Charles Chebat*

Pour d'aucuns le marketing est la source de bien des maux dans la société alors que pour d'autres le marketing est la panacée à beaucoup de maux; quelques fois les mèmes. Le marketing est d'abord une philosophie de gestion des organisations qui reconnait l'importance de la satisfaction de la clientèle. Le concept central du marketing est l'échange. Lorsqu' on "fait" du marketing, on organise ce processus d'échange en taillant l'offre en fonction de la demande, et non vice-versa. Du moins, faut-il tenter de trouver un juste milieu. II s'agit d'un processus systémique oủ le gestionnaire du marketing part de ses connaissances du comportement du consommateur pour préparer une offre intégrée puis se tourne vers le consommateur afin d'en mesurer la satisfaction et de modifier l'offre le cas échéant. Ce processus permet donc d'adopter constamment l'offre face aux besoins changeants de la société.

Le marketing est une science humaine appliquée qui emprunte ses fondements à de nombreuses disciplines. Mais l'intégration de ces diverses influences en rend l'utilisation difficile, il ne faut pas le cacher. Malheureusement, on a souvent eu tendance au cours des dernières années à trop simplifier cette utilisation. Nous allons tenté de voir dans cet article pourquoi l'utilisation du marketing dans divers milieux et en particulier dans le milieu touristique n'a souvent pas répondu aux attentes.

\section{Un apprentissage sommaire}

Quoique le processus d'échange entre les êtres humains soit inhérent à toute organisation, c'est depuis moins d'un demi siècle que le marketing a commencé à être utilisé dans les organisations d'une manière structurée. Le milieu naturel d'origine ètait l'entreprise privee à but lucratif qui fabriquait des produits. Depuis une vingtaine d'années cependant, on a commencé à s'intéresser de façon active au marketing de services. Dans certains milieux comme les banques, les assurances et le tourisme, cet intéreat date

*Pierre Filiatrault et Jean-Charles Chebat sont profes seurs au departement des sciunces administratives de IUUAAM. à peine d'une dizaine d'années. Les organismes publics et des organisations à but non lucratif se sont donc tournés relativement récemment ${ }^{(1)}$ vers le marketing comme moven pour les aider dans la poursuite d'objectifs d'information et de persuasion pour diverses causes lla sécurité routière, le conditionnement physique, la protection de l'environnement, I'information touristique, les campagnes contre la cigarette, etc.).

Nous constatons dans un premier temps que l'apprentissage du milieu touristique (organismes gouvernementaux à tous les palliers, ATR, hôtellerie, etc.) à l'utilisation du marketing ne fait que commencer. Une décennie est une période relativement courte pour développer une culture organisationnelle, des connaissances et une expé rience collective à l'utilisation d'une discipline aussi complexe que le marketing. De plus, non seulement l'expérience collective en marketing touristique est-elle sommaire mais plusieurs individus se sont vus attribuer des responsabilités de marketing sans formation adéquate. Pour pallier cette lacune, des cours de familiarisation ont été organisés dans plusieurs milieux. L'apprentissage résultant de quelques heures de cours et de quelques lectures d'appoint est suffisant pour sensibiliser mais évidemment insuffisant pour développer une expertise. Ainsi, individuellement et collectivement dans le milieu touristique, I"apprentissage au marketing ne fait que commencer.

\section{La nature de I'offre}

Le produit touristique n'est souvent pas un bien mais plutôt un service. II existe de nombreuses différences entre un bien et un service $^{2}$. Un service se différencie d'un bien par son intangibilité et l'absence de transfert de propriété. La nature du concept de distribution diffère aussi dans le cas des services et les connaissances dans le marketing de biens de consommation sont souvent difficiles à transferer ${ }^{(3)}$. En pratique, le marketing d'un service est plus difficile à faire que celui d'un bien. D'ailleurs, les consommateurs semblent éprouver plus de difficultés à évaluer les attributs d'un service que ceux d'un bien ${ }^{(4)}$.
D'autre part, puisque les services ne peuvent être entreposés ou revendus, ils sont souvent consommés en mẻme temps qu'ils sont produits ${ }^{15 t}$, il y a donc production et consommation simultanées d'un service. Dans cette optique, l'effort de marketing devrait être dirigé non seulement vers les consommateurs mais aussi vers les personnes responsables de la production ${ }^{(6)}$. II suffit de penser aux guides touristiques, aux serveuses de restaurant, aux chauffeurs de taxi etc. pour constater qu'il reste encore beaucoup à faire en ce sens dans le milieu touristique.

\section{Les caractéristiques des organisations}

Le développement touristique est la responsabilité des secteurs publics et privés. Le tourisme dans le secteur privé dépend d'entreprises en concurrence qui ont malheureusement quelques fois des objectifs à court terme pressants; ce qui peut résulter en un service de qualité médiocre de la part de certaines entreprises ou encore le produit offert par ces firmes ne s'intègre pas nécessairement avec son environnement. Par contre, en général, l'entreprise privée pour survivre doit se préoccuper de répondre aux besoins des consommateurs et s'assurer de la satisfaction de sa clientèle. Dans ce milieu, plusieurs entreprises sant déjà conscientes de la nécessité du marketing et ont développé une expertise.

D'autre part, les organisations publiques à but non lucratif ont d'importantes responsabilités dans le développement touristique. Les organisations publiques diffèrent grandement des entreprises privees sous plusieurs aspects: les objectifs et politiques, la position souvent monopolistique, la visibilité, les contraintes opérationnelles, les processus administratifs et le scepticisme envers le marketing ${ }^{(7)}$. La raison d'être de l'échange n'est pas toujours clair ${ }^{i p l}$, surtout dans les organisations à but non lucratif. De plus, I'influence des producteurs dans la préparation de l'offre est souvent plus grande que celle des consommateurs ${ }^{\text {al }}$. En d'autres mots, et c'est là une erreur de marketing, les valeurs et besoins des producteurs prévalent parfois sur les besoins des con- 
sommateurs. II faut aussi se rendre à l'évidence qưil n'existe même pas de méca nisme de protection du consommateur dans ce type d'organisation: l'expérience montre qu'on fait peu fréquemment référence aux consommateurs, à leurs intérẻts, à leur protection et à des mécanismes de griefs.

De plus, personne dans l'organisation publique $n^{\prime}$ a la responsabilité intégrée de la clientèle, à partir de l'identification des besoins en passant par la préparation de l'offre et en allant jusqu'a la mesure de satisfaction. Le responsable du marketing dans l'entreprise privée a la difficile tăche d'intégrer toutes les demarches touchant le consomma teur. Dans les organisations publiques ou à but non lucratif, la fonction n'existe tout simplement pas. Le client est officiellement la responsabilité de tous; mais en pratique, personne n'en est réellement responsable. $\mathrm{Ce}$ manque d'intégration du système d'échange dans les diverses organisations touristiques est une première raison fondamentale, à notre opinion, de l'insuccès rencontré dans l'utilisation du marketing dans ces organisations. Tant que dans les divers organismes s'occupant de tourisme, personne $n$ 'aura la responsabilité de la clientèle et l'autorité pour influencer les politiques organisationnelles, il ne se fera pas de marketing dans le secteur touristique.

Une seconde raison expliquant ces insuccès est les difficultés de transferer les concepts et techniques d'un marketing de produits au marketing de services, surtout si ceux-ci sont publics. Nous voulons ici mettre en relief deux obstacles à ce transfert: le premier est inhérent à la nature des services, le second est inhérent au degré d'implication du consommateur.

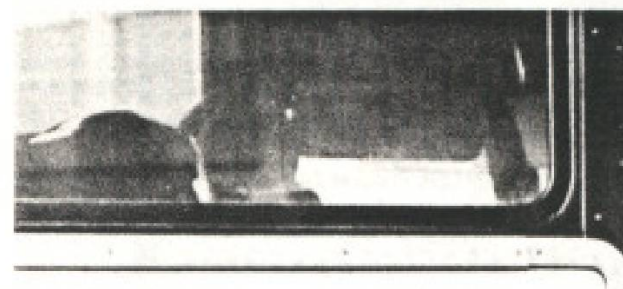

\section{du marketing de services}

Effets organisationnels

Le fait meme que le service soit intangible a pour effet l'absence de stocks lcomment en effet stocker le temps d'interaction du client et du serveur de restaurant?l. En conséquence, l'organisation adopte sa production de services aux fluctuations de la demande dans le temps. L'organisation doit contrôler de façon toute spéciale l'efficacité du personnel en contact. Le personnel employé en période de pointe doit offrir le mème genre et la mềme qualité de services, bien qu'il soit moins "socialisé" à l'entreprise car le personnel en contact est bien plus qu'une production de services, il est aussi aux yeux du client; l'organisation. II doit réa liser les objectifs de l'organisation et fournir des services de façon homogène. La formation du personnel est faite par l'organisation de façon à ce que, quelles que soient les circonstances, le client retrouve le mệme standard de service. Mais, le client lui-même contribue àl'apprentissage du personnel: il $s^{\prime}$ attend aussi à un type et à un niveau de services constants ("tpas de surprise!", est le slogan d'une célèbre chaine d'hôtels qui couvre toute l'Amerique du Nord et l'Europe Occidentale). La variabilité des services affecte le comportement d'achat de services. Ainsi se produisent deux phénomènes convergents: d'une part un freinage dans I'innovation et d'autre part une autosegmentation. En effet, la formule mise sur pied par l'organisation qui réussit se stabilise et finit par passer pour la clé du succès, et le client renforce l'apprentissage de l'organisation en achetant ses services: ce quil stabilise l'évolution de l'organisation. Par ailleurs, compte tenu de la difficulté d'adopter le personnel de contact à plusieurs segments de clientèle, l'organisation fondera sa productivité en concentrant ses efforts sur un petit nombre de clients que son personnel a appris à satisfaire. D'où rigidification du comportement organisationnel.

Implication du consommateur

Dans un excellent article, Rothschild ${ }^{n 0 i}$ souligne que le niveau d'implication du consommateur varie beaucoup selon le type de campagnes promotionnelles publiques versus privees. II soutient que les promotions de produits commerciaux correspondent à un niveau d'implication intermédiaire entre, par exemple, celui de promotions de l'aide aux arts et celui de la sécurité routière. Or cela est fondamental, car la variable "degré d'implication" du consommateur est centrale dans les modèles d'attitudes et de comportements du consommateur. Aussi, le niveau de succès d'une campagne promotionnelle mesuré en termes de changement d'opinion dépend laussil du degré d'implication du consommateur. Le degré d'implication est, selon Rothschild, ou fort ou faible pour la communication publique, mais non moyen comme pour la promotion des produits commerciaux. Or, les décisions reliées au tourisme sont d'implication ou forte ou faible: forte dans le cas d'un voyage à longue distance, coûteux, peut-être hasardeux, avec toute la famille; faible dans le cas d'une excursion du dimanche après-midi. De ces quelques considérations, il résulte que le processus d'apprentissage du consommateur sera bien different selon le niveau d'implication: on peut souligner en particulier que l'activite cognitive len particulier la quantité d'informations recherchées, traitées et memorisées) est très affectée par le niveau d'implication. L'organisation qui se charge de la promotion touristique devrait prendre en compte cette variable pour moduler ses stratégies de communictions.

L'équilibre du marketing mix On confond souvent marketing et publicité par ignorance. Or, la publicité n'est qu'une des composantes de la communication marketing lon y retrouve aussi la promotion des ventes, les relations publiques et la communication personnelle ou ventesl, et la com: munication n'est qu'un ellement du marketing mix. Le marketing mix est composé, en plus de la communication du produit lou servicel, du prix et de la distribution. Ce mix se justifie par le concept économique suivant: un effort réparti sur quatre variables provoquera plus d'effets que le même effort, de même intensité sur une seule variable. C'est l'évidence des rendements décroissants. II est indéniable que l"équilibre entre chacune des composantes du marketing mix est difficile à définir.

La première composante est le produit touristique qui prend plus souvent la forme de services que de biens. L'importance du développement d'un produit touristique gênérique a déjà été soulignèe par Demers ${ }^{111}$. L'adaptation et la préparation du produit se font ensuite à partir d'études
L'effort de marketing devrait être dirigé non seulement vers les consommateurs mais aussí vers les personnes responsables de la production. 
de comportement du consommateui et d'évaluation de l'appréciation de ce produit. Preparer un produit sans définition prealable de ses attributs en fonction d'un marche cible est une erreur de marketing.

Pour préparer un produit, il faut faire la synthèse de l'homogénéité et de l'hétérogénéité de la clientele et des ressources; ce qui est difficile en milieu touristique. Le pou. voir des responsables du tourisme dans le secteur public est limité. Or, une partie du produit touristique est la responsabilité de I'entreprise privée. Chaque entreprise assurant son développement sans nécessairement l'intégrer à un produit touristique régional ou national. Par contre, dans l'entreprise privée, on a plus tendance à s'occuper des besoins de la clientèle que dans le secteur public. Parfois même, I'entreprise privée tombe dans l'excès contraire ${ }^{(12)}$. Trop répondre aux besoins peut résulter en des abus mettant en péril la créatitvité, la mise en valeur du patrimoine, l'esthétique et même la protection de l'environnement. Ainsi, l'intégration en matière touristique est en partie ou en tout la responsabilité du secteur public qui $n$ 'a pas toujours en main les outils pour assurer l'intégration du produit touristique et la complémentarité de ses composantes. Le problème devient plus compliqué si on considère les disparités régionales.

Le prix est la deuxième composante du mix; cette variable est imposée en partie par le contexte socio-économique local. La fixation des prix ne devrait cependant pas se faire uniquement sur une base comparative locale mais plutôt sur une base comparative internationale. Si le prix est trop élevé, la région concernée y perdra de trois facons: les gens du milieu ne voyageront plus dans la région, ou encore ils iront à l'extérieur et enfin, les touristes étrangers trouveront de meilleures aubaines ailleurs et ne viendront tout simplement pas dans la région. La troisième composante est la distribution ou l'accessibilité. La distribution consiste à structurer des réseaux d'intermédiaires pour aider à activer la demande alors que l'accessibilité a pour raison d'être de faciliter aux touristes son intégration. Là aussi, nous nous retrouvons avec des dualités de responsàbilites. L'accueil aux aéroports, l'organisation du transport terrestre, la signalisation sont la responsabilité du secteur public. Alors que d'autres volets, comme le taxi et I'autocar sont des responsabilités privées. II faut bien l'admettre que le premier contact d'un touriste étranger soit par le transport routier (et la signalisation...) ou par le transport aérien (Mirabel, Dorval, les taxis, les autocars, etc.) puisse donner une image négative du Québec et que cet aspect du marketing a êté nègligé.

Et, il reste la difficile question des communications car on ne mise pas toujours sur la bonne composante du mix de communica- tion et le cadre théorique retenu n'est pas nécessairement le bon non plus. Nous nous tournerons maintenant vers cet aspect non s'en rappeler qu'un élement fondamental de la gestion marketing est l'orchestration de la communication aux autres composantes du marketing mix.

La communication provenant de mass media est censée être médiatisée par le réseau de communication interpersonnelle avant d'atteindre le récepteur (c'est le modêle dit du "two-step-flow"). La réalité est en fait plus complexe et moins malleable. Tout particulièrement si l'objet de la communication est d'implication forte. Depuis Klapper ${ }^{[134}$ et Festinger ${ }^{144}$, on sait mieux comment les changements d'attitudes génèrent des changements de comportements. Si les média peuvent provoquer des changements d'attitudes, ce sont les pairs, les semblables, les gens avec qui le récepteur est en contact qui creent, stabilisent ou éliminent les changements de comportements. C'est vrai des campagnes sur l'alcoolisme, la médecine préventive, la sécurité routière ou les organismes de charité et nous ệmettons l'hypothèse que le phénomène est vrai pour le secteur touristique. La publicité est une condition nécessaire au changement mais elle doit tenir compte, non pas seulement comme on le fait trop souvent, de l'individu mais aussi de son réseau de communication interpersonnelle. En particulier, les décisions de vacances (où, combien, quand, etc...) impliquent un niveau minimal de consensus du couple, de la famille ${ }^{(15)}$ ou dans l'organisation. Se préoccuper du seul chef de famille ou du representant de l'organisation est une aberration méthodologique, d'abord, stratégique ensuite. Le résultat en est trop souvent l'échec commercial.

\section{Conclusion}

Le marketing est une science humaine appliquée. Collectivement et individuellement I'apprentissage au marketing en milieu touristique au Québec ne fait que commencer. Nous avons vu que le secteur public a une grande responsabilite dans le développement touristique et que ce secteur aurait tendance à négliger les consommateurs alors que dans le secteur privé on porterait moins d'attention à la qualité et à l'intégration à I'environnement. Par contre, dans l'entreprise privé, une personne est responsable d'intégrer toutes les facettes des relations avec la clientèle; dans le secteur public cette responsabilité est assignée à tous ou presque, mais à personne en particulier. Les raisons de l'insuccès du marketing touristique ont été attribuées à l'absence de responsabilité intégrée de la clientèle êt à la difficulté du transfert de concept considérant en particulier les effets organisationnels et le niveau d'implication du consommateur.

Nous avons aussi parié de la complexité d'orchestrer le marketing mix du tourisme.
La gestion du marketing touristique au niveau national est particulièrement difficile à cause de l'hétérégoneité des divers produits. Les problèmes de prix et de distribution (signalisation, taxis, etc.) ont été soulignés. II a enfin été mentionné que la valeur de la communication personnelle auprès des consommateurs est souvent sous-estimée par rapport â l'influence du réseau de communication interpersonnelle.

Le marketing peut aider au développement touristique si la formation en marketing des intervenants est améliorée, si l'entreprise privée apprend à se mieux concerter pour per: mettre une meilleure intégration de son produit, si les organisations publiques accordent plus d'importance à leurs clientèles, si le prix devient plus concurrentiel, si l'acces sibilité est facilitée et si la communication est rendue plus efficace. Enfin, l'apport du marketing au tourisme sera d'autant plus marqué que la recherche sur les effets orga nisationnels du marketing de services et sur le comportement du consommateur du produit touristique aura été féconde. f

\section{Références}

(1) KOTLER, Philip, Marketing for Nonprofit Orga: nizations, Prentice-Hall, Englewoof Clifts. 1982 N.J.

(2) LOVELOCK. Christopher H. at WEINBERG Charles B., Contrasting Private and Public Sector Marketing. Combined Proceedings AMA. Chicago III, p. 242.247

(3) LOVELOCK, Christopher H. Services Marke ting. Prentice Hall, Englewood-Cliffs, 1984, N.J.

(4) JOHNSON. Eugene M. Seling of Serwices in Handbook of Modern Marketing. Victor P Buell ed. McGraw-Hill, 1970. New York.

(5) RATHMELL John M. Marketing in the Service Sector. Winthrop Pub., 1974, Cambridge Mass.

(16) Eiglef, Pierre. LANGEARD, Eric, LOVELOCK, Christopher H., BATESON, John E. et YOUNG, Robert F. Marketing Consumer Services: New Insights, Marketing Science institute, 1977, Cam bridge, Mass.

(7) RITCHIE, J.A. et LA BRECQUE, Rioger J., Marke fing Research and Public Policy: A functiona Perspective. Journal of Marketing. 1975, (July)! p. $12-19$.

(B) KOTLER, Philip et ZALTMAN, Geraid, Social Mar keting: An Approsch to Planned Social Change. Journal of Marketing, 1971, Jully, D. 3.12

(9) ETGA, Michat el RATCHFORD. Brian T., Mar kefing Management and Marketing Concepr: Thew Conflict in Non Profit Organizations. Com. bined Proceedings AMA, 1974 . Chicago 111. p. 258-261

(10) ROTHSCHILD, Michael L. Marketing communte carion in Nonbusiness Situstions on Why /t's Harder to Sell Brotherhood Like Soap. Journal of Marketing. 1979. (Spring), p. 11-20

(11) DEMERS, Jacques, Le tourisme en péril, Nouvelle Optique, 1983. Montréal.

(12) GENNET, Roger et COOPER, Robert, The Misuse of Marketing: An American Tragedy. Business Horizons, 1981. Vol. 24, p. 51-61.

(13) KLAPPER, J. The Effects of Mass Communication. The Free Press, 1960, New York.

(14) FESTINGER, Leon, Behavioral Support for Opo nion Change. Public Opinion Quaterly, 1964. Vol. $28, \mathrm{p} .404-417$.

(15) FILIATRAuLT, Plente et RITCHIE, Brent J.A. Family Vacation Decision-Making - A Replication and Extensoon. Journal of Travel Research. Vol. XVIII, NNo 4, 1980, (Spring), p. 3.14 